\title{
The Pursuit Of Climate Protection And The Uneven Global Distribution of Clean Development Mechanism (CDM) Projects: Lesson From Least Developed Countries (LDCS)
}

\author{
Philip Agbonifo ${ }^{1} \mathrm{PhD}$
}

\begin{abstract}
In this modern era of climate protection, the need for climate justice in the distribution of projects cannot be neglected or postponed. While the pursuit of climate and environmental protection, sustainable development and green technologies have become increasingly topical, issues surrounding the sustainability of the Least Developed Countries (LDCs) within the context of climate protection are being unwittingly developed and ignored. Thus, this article offers a critique of the uneven distribution of Clean Development Mechanism (CDM) projects. While most projects have gone to the emerging countries of China, India, Brazil and Mexico with opportunities for investment, unfortunately very little projects were established in the LDCs of Africa and Middle East. This prompts the question whether the CDM is fulfilling its climate protection goal, or does it currently deliver its sustainable development claim in the LDCs? Only a relatively few countries that are attractive destinations for foreign direct investment have benefited immensely from CDM projects. Consequently, this paper looks into the distribution of CDM project activities among developing countries, justification for the distribution, and examines what can be done to ensure equity among developing countries concerning the harnessing of the benefits of the CDM.
\end{abstract}

Keywords: Least Developed Countries (LDCs); Clean Development Mechanism (CDM); sustainable development; climate protection; uneven project distribution.

\section{Introduction}

Clean Development Mechanism (CDM) is a project based "flexible mechanisms", largely regarded as a climate protection instrument, the first and by far the largest carbon offset instrument of its kind, established to achieve cost effective solutions to mitigate climate change. The mechanism is meant to bring about a range of direct and indirect economic and social effects in the project country, which aims to contribute to sustainable development and provides a standardised environmental protection and development instrument through Certified Emission Reduction (CER) credits (Shishlov and Bellassen 2012),. It allows industrialised nations with an obligation to protect the environment and to achieve their reduction targets by supporting appropriate projects to limit climate change in developing countries, amongst other things (UNFCC 2011). Similarly, Lutken (2011) argued that the cost of environmental protection varies considerably from region to region, as a result of differences in, for example, energy sources, energy efficiency and waste management. Furthermore, he maintained that the world has embraced the mechanism - with all its flaws and 
shortcomings as an instrument for international cooperation on sustainable development and environmental protection. This development, therefore, suggests that the CDM continues to appear to sustain uneven development patterns overlooking those most in need (Woods et al 2015).

The main major objective of the CDM is to assist weak economies to solve their environmental problem, and this argument has attracted much attention in the literature. Interestingly, researchers have focused on reviews of the CDM projects activities and trends, theoretical prediction of factors affecting project distribution, analyses of the technology transfer and development impacts of CDM projects. Furthermore, the World Bank and the UNFCCC publishes annual review of activity in the carbon market that includes a breakdown of CDM project buyers, host countries, and project types. There are other review which focus on the market potential for CDM projects, with particular reference to the dominance of China, India, Latin America and other emerging markets (Olsen and Fenhann 2008; Watson and Fankhauser 2009). Even though CDM has made notable contribution to climate change, however, some literature (Silayan 2005; Boyd et al 2009; Olawuyi 2010; Michaelowa et al 2014) have documented the lack of CDM projects in LDCs, however, they offer little in the way of potential solution.

The policy document and academic literature argument suggest that the CDM as presently constituted does not have the potential to influence development in the LDCs, particularly in Sub-Sahara Africa and the Middle East (Silayan 2005; Michaelowa et al 2014; Wood et al 2015). The measure of hope and progress accruing from CDM projects across the world have gone to the emerging countries of China, India, Brazil and Mexico with very little opportunities for investment in the LDCs. In his article on the problem of justice in the global climate markets, Sovacool (2011) argued that the critical questions about justice in the CDM is that they tend to benefit the countries that are most industrialised (or industrialising), not actually those most in need. The CDM is a competitive mechanism and has continuously favoured the industrialised countries and that explains why the strong and emerging economies such as Brazil, China and India has continued to attract investors as oppose to the poorest countries in Africa and Middle East that are in dire need of projects but lack such capacity. Because investors have quickly exploited the best location and most profitable projects, thus the CDM has further created the problem of inequalities between countries.

The inclusion of more developed nations in the climate change process evidently revealed that current trends of the CDM show a clustering of projects towards a few larger developing countries. Ideally, it is the universal concern that equitable distribution of projects is achieved among developing countries. CDM as a market mechanism implies that investors in the market must pursue an optimal investment portfolio to maximize profit and minimize cost (Silayan 2005), unfortunately, this scenario dictates the concentration of investment to only a few countries. Thus, Lutken (2011) argued that central to the debate of CDM in LDCs is the problem which lies in the use of a marketbased mechanism in a climate change framework that espouses equity among different parties.

With a sound theoretical argument left alone to market forces for a single commodity, there will always be winners and losers in the climate protection market of the CDM. The winners are countries like India and China whose national structures are geared 
towards the development of CDM projects while the losers are Africa and the Middle East countries. The winner is reaping the full benefits of sustainable development through the transfer of technologies, on the other hand are those who are unable to provide enabling environment for investment to strive which are the major losers. Based on the early warning on the exclusion of the LDCs from CDM, Silayan (2005) maintained that enforcing redistribution of projects among developing nations need not take a complete rethinking as equitable distribution of projects is possible within the current structure of the CDM framework.

To this end, Olawuyi (2010) argued that if truly the CDM was adopted to assist weak economies as defined in Article 12 of the Kyoto Protocol in solving their environmental problems, it is ironical that these LDCs, despite their apparent weaknesses have to engage in an unending and competitive struggle to capture this assistance. Distinct conditions such as regulation, security, infrastructure, capacity building and country specific barriers among developing countries have led to different implementation circumstances. The protocol allow developed country with the freedom to choose which developing country it wishes to invest in and to choose the least investment risks in terms of legal framework, investment climate and high mitigation potential for their projects. This development prompted the argument by Silayan (2006) that applying the economic theory of emissions trade on an international scale involving a variety of nations in varying stages of development manifests a complex equity problem that has become the bane of the CDM. The imposition of a model of a market based mechanism on countries with different levels of competitiveness creates a trading environment conducive for imperfect competition whereby only a few nations benefit, and overall social welfare decreases. This deviate sharply from the argument that investors benefit from wider distribution of projects which also expands the scope of the supply of emission reduction credits, thereby reducing the developed countries cost of compliance with the Kyoto Protocol on climate change.

\section{Economic Argument of CDM}

The major key economic benefits of CDM hinges around sustainable development which also include growth in green energy and development, climate protection projects, mitigation of environmental degradation and employment opportunities (Boyd et al 2009; Alexeew et al 2010). Similarly, Gupta (2012) argued that CDM did not only support basic development needs of developing countries but has also contributed immensely to technology transfer in no small measure. He maintained that carbon finance market through sales of CERs have increased significantly from US $\$ 2,634$ million in 2005 to US\$32.79 billion in 2008. Other academic argument (Olsen and Fenhann 2008) argued that sustainable development contributions are predominantly social, closely followed by economic and finally environmental. Watson and Fankhauser (2009) opined that sustainable development is largely interpreted as a three dimensional concept which encompasses environmental, social and economic components. In addition, they argued that from a pragmatic approach a clear distinction is made between economic growth and development and sustainable development which are often times, commonly grouped together. They opined that economic growth and 
development is used to refer to project benefits that are immediate, but have not necessarily sustained; employment, further income opportunities for communities local to projects, and short term livelihood improvements.

Advocates of CDM draw on theories of innovation, technology transfer and energy transitions to argue that it can help to spark innovation in the developing world (Liverman and Boyd 2008). The mechanism builds on the institutional experience established by the UNFCCC to connect the economic, scientific, financial, developmental and political perspectives of the climate change liaising with national government (Byigero et al 2010). The scientific literature on the CDM often focuses on the requirement of delivering cost efficient emission reduction that is real, measurable, and additional, and contribute to the sustainable development of the host community (Boyd et al 2009; Alexeew et al 2010; Olsen 2012). Other authors have explicitly studied the environmental integrity of the CDM (Olsen and Fenhann; Watson and Fankhauser 2009; Gupta 2012), but question the rigorous debate that many registered domestic projects would have happened anyway without the CDM (Liverman and Boyd 2008). Furthermore, some studies argued that $\mathrm{CDM}$ has been more effective in reducing mitigation costs for industrialised nations than in contributing to sustainability in developing countries (Olsen and Fenhann 2008).

On the difficulties in defining sustainable development, (Figueres and Streck 2009) argued that $\mathrm{CDM}$ is unable to address all the issues relating to environmental protection, however, it remains a mechanism in its adolescence trying to find its rightful place in the scheme of things within the international climate change concept. However, the CDM has attracted some criticism, questioning its environmental effectiveness, economic efficiency and regulatory functions (Liverman and Boyd 2008). Just as Henry Ford continues to remind his employees that "everything can be done better than it is", the focus is how to build on the existing CDM to create much greater participation and improvement, from a project based level of a sector or programme based level (Ellis and Kamel 2007; Olsen and Fenhann 2008).

The evidence from literature has demonstrated that while one of the two requirements of CDM is to improve development levels in host countries (Boyd et al 2008), unfortunately, many researchers have discussed the lack of development impacts of projects and transfer of technology (Silayan 2005; Watson and Fankhauser 2009). Ultimately, the confluence of the CDM trends which is a preference for industrializing economies and depending on the Western firms for technology transfer with lack of optimal sites, meant that carbon markets rarely, if ever, reduce poverty and meet millennium development goals (Michaelowa et al 2014). Sutter and Parrano (2007) carried out a study in 16 registered CDM projects in Brazil, Honduras, India, South Korea, Bhutan, Bolivia, China, Chile, and South Africa (involving a variety of projects, including landfill gas recovery, construction of hydroelectric facilities and wind farms, electricity generation from crop residues, natural gas fuel switching, weatherization and housing upgrades) and looked at their propensity to generate jobs, reduce poverty, improve local air quality, and reduce emissions. They discovered from their studies that none of the projects scored high on meeting development goals and reducing emissions simultaneously, finding that $95 \%$ add little value for sustainable development in the project locations. The situation is not different from India, where CDM project which 
was meant to contribute to poverty alleviation in rural areas, revealed that landless labourers did not benefit, instead, many CDM projects increased prices in some agricultural commodities. The employment benefits for poor and unskilled labourers were extremely limited, projects were generally concentrated in areas with far-above average incomes, and benefits tended to accrue to a limited number of elites. Similarly, in Nigeria, the CDM project in Kwale and Ovade-Ogharafe for the recovery of associated gas for electricity generation presents a huge question mark. All the potential socioeconomic benefits and poverty alleviation to the host communities appear to be rhetoric. Only a relatively few neighbouring community where the CDM project is located have access to electricity supplies on a partial basis. The projected electricity generated from the CDM projects benefiting primarily the wealthier Nigerian urban communities at the expense of the majority of rural poor communities.

In addition, Olsen (2007) carried out a meta-analysis of 19 studies on the CDM and actually discovered a trade off between the CDM target of supplying cheap emissions credits and the promotion of sustainable development, with the former taking priority over the latter. Olsen concluded that the CDM did not drive sustainable development goals, did not promote renewable energy projects, privileged projects that brought in investment (rather than those that promoted development), and did not benefit poor households. Also, Alexeew (2010) argued that in some cases, CDM projects actually worsened sustainable development goals of the communities by interfering with land use practices, increasing the price of fuel wood, or forcibly relocating communities. Similarly, Michaelowa and Michaelowa (2007) surveyed climate change projects being undertaken in the developing world and concluded that they resulted in only "limited" benefits at meeting millennium development goals. They noted that projects addressing poverty were very rare and that even small renewable energy projects in rural areas usually benefitted rich farmers and the urban population at the expense of the very poor.

\section{Regional Distribution of CDM Projects}

Some literature (Lutken 2011; Shishlov and Bellassen 2012; Wood et al 2014) argued that the CDM is inherently unsuitable for the LDCs due to lack of appropriate regulation, infrastructure and investment climate. These factors attract more CDM projects to countries like China and India compare to the LDCs of Africa and Middle East. These challenges can be tackled through preferential treatment, country eligibility screening, import quotas and discounting, essentially as an instrument to influence the more advanced developing countries to move to a more ambitious abatement action for projects in the LDCs.

Despite its flaws and challenges, CDM has been the largest carbon offsetting mechanism in the world which has grown rapidly in the last couple of years. Shishlov and Bellassen (2012) argued that CERs supply is highly concentrated with 93\% of all issued credits coming from just 5 largest CDM countries: China, India, South Korea, Brazil and Mexico, while Africa countries account for less than $2 \%$. These countries have become the CDM stars because they possess the key factors such as strong institutional capacity and favourable investment climate influencing the CDM attractiveness of the host countries. Furthermore, some developing countries like China pursue an 
increasingly large number of unilateral CDM projects without foreign direct investment as their need for new technology and foreign investment is lower than LDCs (Shishlov and Bellassen 2012). It is also argued that the CDM potentials for the LDCs are significantly lower due to lower absolute levels of emissions and low carbon intensity of their economies and technological barriers. LDCs often suffer certain barriers such as well functioning institutions, favourable investment climate and infrastructure that is a prerequisite for investors.

$\mathrm{CDM}$ as a market mechanism cannot in itself guarantee an equitable distribution of projects among developing countries under the present scenario, because of differences in regulation, infrastructure, security and investment climate between countries and regions. The industrialised countries recognised that under the present scenario that the CDM cannot lead to an equitable distribution and there is the need to look elsewhere for alternatives to enforce distribution of projects. Geographically, the distribution of CDM projects has so far not been very equitable (Lutken 2011), and there is no regulation to find a solution that addresses both equity and cost effective solution to CDM project distribution. A limited number of countries including China, India, Brazil and Mexico have captured the largest share of the global CDM project portfolio (Gupta 2012). Specific regions in the developing world, namely Sub-Saharan Africa, have been largely bypassed by the CDM market and are struggling to attract a decent number of CDM projects (Lutken 2011). In fact, of the total 2,647 projects, only 33 projects are in SubSaharan Africa, and 21 of these are actually in South Africa, making the distribution even more skewed (UNEP 2007). More specifically, UNFCCC (2014) noted that of the 7,426 registered CDM project's distribution by host part as at January 2014 revealed Asia \& Pacific with 84.2\%, Latin America \& Caribbean with $12.8 \%$, Africa $2.4 \%$ and Economies in transition $0.6 \%$. In addition, of the total 178 projects in African, 54 representing 30\% are located in South Africa alone while other African countries' shares 124 (UNFCCC 2014).

Gupta (2012) further emphasised that there is an uneven regional distribution of projects as more than three quarters of current CDM projects around the world are based in just four countries. Similarly, Hong et al (2011:1695) has argued that "as at 2011 China has attracted the lion's share of CDM investment with $45 \%$ of the world projects and account for 63\% of the global annual CERs". More importantly, while CDM is a success story in China, it is not the case with Nigeria with just twelve projects which poses serious question about the future prospect of CDM in the LDCs where it is marginally low. This is congruent to the views expressed by Lutken (2011); Gupta (2012) that the number of registered projects in LDCs is almost negligible in comparison with the rest of the world.

The table below shows the top CDM 10 countries having the highest numbers of registered CDM projects across the world.

Table 1: Top 10 CDM Registered Projects Countries in the World

\begin{tabular}{|l|l|l|l|}
\hline Country & $\begin{array}{l}\text { No. of Registered } \\
\text { CDM Projects }\end{array}$ & $\begin{array}{l}\text { Estimated Emission } \\
\text { Reductions }\left(\mathbf{C 0}_{2} \mathbf{e} / \text { year }\right)\end{array}$ & $\begin{array}{l}\text { Average Project } \\
\text { Size }\left(\mathbf{C 0} \mathbf{0}_{2} \mathbf{e} / \text { year}\right)\end{array}$ \\
\hline China & 1,858 & $367,754,013$ & 197,930 \\
\hline
\end{tabular}




\begin{tabular}{|l|l|l|l|}
\hline India & 805 & $67,474,383$ & 83,819 \\
\hline Brazil & 205 & $24,175,021$ & 117,927 \\
\hline Mexico & 140 & $12,520,350$ & 89,431 \\
\hline Malaysia & 110 & $6,293,316$ & 57,212 \\
\hline Vietnam & 90 & $5,410,200$ & 60,114 \\
\hline Indonesia & 80 & $8,308,580$ & 103,857 \\
\hline Thailand & 67 & $3,541,395$ & 52,857 \\
\hline Republic of Korea & 63 & $18,187,041$ & 288,683 \\
\hline Philippines & 57 & $2,238,466$ & 39,271 \\
\hline All other countries & $\mathbf{4 7 4}$ & $\mathbf{6 7 , 5 2 0 . 1 6 7}$ & $\mathbf{1 4 2 , 4 4 8}$ \\
\hline
\end{tabular}

Source: UNFCCC (2012)

It should be noted that the assessment on the table above is based on 3,949 registered projects or undergoing registration as at June 2012.

Advocates of the CDM draw on theories of innovation and energy transition in the developing world. But, there are uneven project distributions across countries and regions, which raises serious question about the equitable distribution of projects across the developing world (Ellis and Kamel 2007). CDM projects are lip services of Western countries to climate change and green economy concept because Nigeria has gained little from technology transfer to kick start development activities and eradicate the problem of environmental degradation. According to Boyd et al (2009) Africa and Middle East have 43 projects equivalent of 3\%, compared to Asia and Pacific with 923 projects representing 67\% and Latin America 394, equivalent of 29\%. Liverman and Boyd (2008) termed this as 'distributive justice arguments' due to the unequal pattern of CDM projects with a bias to larger developing countries such as India, China, Brazil and Mexico.

Table 2: Regional Distribution of Registered Global CDM Projects as at December 31, 2010

\begin{tabular}{|l|l|l|l|}
\hline S/No & Region & No. Of Projects & \% Total \\
\hline 1 & Africa & 39 & 1.44 \\
\hline 2 & East Asia and the Pacific & 1,479 & 54.73 \\
\hline 3 & Europe and Central Asia & 20 & 0.74 \\
\hline 4 & Latin America and the Caribbean & 496 & 18.34 \\
\hline 5 & Middle East and North Africa & 49 & 1.81 \\
\hline 6 & South Asia & 620 & 22.94 \\
\hline & TOTAL & $\mathbf{2 , 7 0 3}$ & $\mathbf{1 0 0}$ \\
\hline
\end{tabular}

Source: Gupta (2012)

This lopsided development has enabled over $80 \%$ of CDM projects to be clustered in Asian countries, while Africa accounts for less than $2 \%$ of the entire CDM (Olawuyi 2010). In particular, out of the 11,000 projects submitted by January $2014,70 \%$ are hosted by China and India alone (Michaelowa et al 2014). This creates a trading environment conducive for imperfect competition (Olawuyi 2010), and many poor developing countries would be caught up in a fierce contest of winning CDM project at all cost. This makes the concept of sustainable development, the primary aim of the CDM more strikingly difficult to achieve. Similarly, Gupta (2005) maintained that the 
argument that CDM must assist developing countries for sustainable development has got lost, and financially strapped poor countries are forced into a mindless competition to facilitate the selling of credits, cheaply and as fast as possible.

The trend in CDM climate change philosophy which allows developed countries to neglect weaker developing nations from project participation may not on this score, represent the best mitigation route to the global community (Shishlov and Bellassen 2012; Michaelowa 2014). Significantly, it also, may not win its war against environmental integrity if all nations no matter how poor or small are now involved. Therefore, in an attempt to attract all forms of CDM investments, many LDCs have deliberately lowered their sustainability standard criteria which raises the question whether these countries will not again turn to dumping grounds for cheap and dirty technologies masquerading as CDM projects. Consequently, Olawuyi (2010) argued that the imperfect competition for CDM projects has brought about new questions on how sustainable the CDM will be at the end of the day for developing countries that are now opening their doors to any form of project.

Lutken (2011) has always advocated levelling the playing field of CDM project worldwide to avoid carbon leakage of the CDM. In his article, indexing CDM distribution, he argued that while projects have sprung up in Asia and Latin America from the very early day, regrettably Africa was lagging far behind. Thus, LDCs have yet to experience the advantages of embarking on the CDM project development. This is further supported by Boyd et al (2009:821) that CDM is now widely viewed as an imperfect but useful approach to encourage the development of emission-reduction projects in developing countries. The Nigeria scenario with limited CDM project and its history as the second worst gas flaring country behind Russia Federation paint a picture of an established shortcoming, flaws and challenges in the CDM geographical distribution of projects.

\section{Policy Barriers and Key Challenges of CDM in LDCs}

The CDM project has become an instrument of foreign policy that creates new structural dependencies with the expansion of resources and atmospheric capacity inimical to LDCs. Olsen and Fenham (2008); Olawuyi (2010) noted that estimates have clearly shown that LDCs have failed to live up to their emission reduction credit potentials within its confines. In particular, African countries have more than 3,200 clean energy projects and 740 million tons of GHG reduction per year, unfortunately, the continent account for a meagre $2 \%$ share of global CDM pipeline. There is an increasing sense of urgency about the delivery of clean development projects in the LDCs. This necessitate that appropriate governance is needed to overcome the problem of climate change, energy poverty and energy security. In addition, if carbon and energy finance of the CDM is to realise its potential to tackle any forms of energy poverty, it has to serve the needs of the poorest people like the Africa and Middle East. Accordingly, Asia and Latin America hold $97 \%$ of all project activities in the CDM virtually leaving out countries in Africa and the Middle East disproportionately.

Barriers to CDM varies between countries and a combination of factors is needed to drive growth in a country's CDM activity which include the presence of attractive CDM 
opportunities, a positive investment climate, enabling policy and legislative framework (UNEP 2007). Other challenges such as national level barriers as electricity regulations which constrain projects, institutional capability or lack of awareness about the CDM potential can dampen interest in CDM projects. In fact, there is also the question of availability of underlying project finance that render the performance of the project uncertain and uncertainty regarding project eligibility. Furthermore, politics and institutions are very important to the CDM, and investors seek opportunities in countries for their investment to facilitate and bring sustainable development benefits. This is because the governance of $\mathrm{CDM}$ has become more exclusive, inequitable and non transparent in the distribution of projects globally.

One of the key challenges facing LDCs interested in participating in the CDM market is the complexity of modalities and procedures. This has resulted in some CDM stakeholders in LDCs presenting poorly designed CDM projects that eventually get rejected. Additionally, some LDCs have not been able to participate in the CDM primarily due to lack of national CDM expertise and/or the appropriate institutional setup necessary for the assessment and approval of CDM projects (UNEP 2007). The complexity and modalities of procedures are a major challenge facing developing countries interested in participating in the CDM which often result in poorly designed projects that are eventually rejected. In most cases, some LDCs are not able to participate in the CDM due to lack of national CDM expertise and/or the appropriate institutional framework to facilitate assessment and approval of CDM projects (UNEP 2007).

Access to predictable flows of finance is an additional barrier facing CDM project developers in many countries, partially due to lack of CDM knowledge among LDCs financial intermediaries. Consequently, there is a clear need for human and institutional capacity building on finance within the area of CDM in many LDCs. Moreover, there is currently no clear cut internationally recognised guidelines on the allocation of capacity development funds, which has resulted in some countries receiving more funding while others receive no funding at all. The expectation are that CDM in LDCs demonstrate the basis of internal rate of return (IRR) that is potentially viable enough to lure investors, because the hurdle of convention finance in the name of CERs is not a panacea for projects that make no financial sense at all. Whereas, the lack of access to conventional finance is one of the bane of the development of the CDM in Sub Sahara Africa and is also attributed to economic viability of most ideas that are not bankable projects.

What is significantly important is the argument that the major barriers to CDM in LDCs are lack of viable project with respect to the underlying business maths and adequate knowledge base. There is little opportunity for individuals or communities to understand or engage with effect of the project on the community. Investor is not attracted by the mere great idea that it is a CDM without considering the basic study done on viability of the project. Although so many projects opportunities have been identified in the LDCs, particularly in the sub Sahara Africa, however, there are complexity associated with CDM projects registrations, validation and implementation. There are other problems such as capacity building, regulation, country specific barriers to CDM development, access to basic infrastructure, security and government bottlenecks that are critical to project implementation. 
There is lack of awareness and collaboration on the part of most financial institutions on what $\mathrm{CDM}$ is all about for which foreign investment in any project is critical. The absence of commitment and understanding of such project by the locals is evident in the case of Africa and the LDCs. Institutional developers and policy makers have the potential to develop carbon assets but this has to be done in collaboration with the local organisations which have been partly responsible for the uneven distribution of projects in the LDCs.

Moreover, most of the LDCs are perceived to be risky for investment purposes by the international investors, which are prime important consideration in CDM development barriers. The Niger Delta region of Nigeria noted for activities of unrest, violent conflicts and kidnapping for ransom is a prime example of deprived investment in the LDCs. Although the risk in CDM revenue accounts for only $20 \%$ of the overall revenue, the expected growth in CDM development is due to perceived risk by most institutional investors.

The non-existence of baseline data which is required for effective participation in the CDM market in LCDs can be seen as an unofficial barrier to participating in the CDM. Smaller and poorer developing countries like Africa and the Middle East, many of whom are categorised as LDCs, have difficulty providing existing baseline data. Without baseline data, a baseline scenario will be very difficult to conceive, objectively proving that project additionality is almost impossible (Silanya 2005). Moreover, the structural implementation which allows the CDM to be concentrated in fewer countries with much of the LCDs unable to participate is merely creating inequality among developing countries. The implementation conditions outlined in the CDM impede many of the poorer developing countries in the CDM participation and that explains why some countries are better than others in attracting and initiating CDM projects.

\section{The Mechanism of Dispossession and Inequity}

The structure of the CDM encourages the capitalistic model of dispassion and an instrument to expand the unintended capitalist globalisation whereby the wealthiest nations continue to accumulate by dispossessing the excluded LDCs. The CDM may appear to be pursuing a regime of expanding accumulation through the carbon dump for the developing countries (Silanya 2005), while the industrialised countries pay the bill for not having the right to pollute.

In the name of sustainable development, there appear imposed development hegemony and capitalistic values that allow the gain of CDM and the transfer of large scale clean technologies which serve the powerful global interests. This undermined the traditional ways for sustaining local and indigenous people's livelihoods, which are act as an invaluable source of ecological sustainable alternatives. Thus, the global policies that intensify inequalities in the distribution of CDM projects, social exclusion and justice and accumulation by dispossession practices are false market solutions for development that cannot be sustained for too long. Unfortunately, the incorporation of the environmental policy framework into the heart of liberal market institutions, like the World Bank enables a more rooted institutionalisation of green capitalism. More importantly, is the argument that the CDM hegemonic discourse attempt to persuade the idea that a green 
capitalist economy is ideal for economic growth that could achieve the miracle of sustainable development and continuous growth.

This is further compounded by the problem of discrepancies in the level of local capacity for the implementation of the CDM, leading to the clustering of projects in a few countries where seven out of 135 developing nations hold $68 \%$ of all CDM projects. These inequalities have been augmented by current patterns of capacity building fund distribution which has been disproportionately allocated to larger developing nations in Asia and Latin America at the detriment of the LDCs.

Such factors as economic benefits, efficient energy development and utilisation, improvement in the environment, transfer of technology and emission reduction are relevant in the Nigeria context. There are however some key economic benefit of the CDM which include growth in green energy in developing countries, engage all stakeholders in environmental protection projects, give adequate guidance to developing countries on how to mitigate environmental degradation. It also promotes investment in green technology (low carbon economy), developed carbon trading market, create awareness on climate change and provide funds and modern innovative technologies necessary for projects with capacity to combat environmental degradation.

\section{The Nigeria Scenario of the LDCs}

Undoubtedly, CDM has emerged as one of the important tools to sustainable development which has helped to spur development in developing countries, but its relevance to pace of environmental degradation in Nigeria cannot be fathom. As with most LDCs, the CDM contribution to sustainable development in Nigeria is marginal and merely rhetoric based on project design documents and existing projects. For example, employment generation from CDM projects to teeming unemployed youths of host oil and gas producing communities in the Niger Delta remain negligible. It remains to be seen whether any connection really exists given that the oil and gas industry is primarily driven by technology and highly capital intensive, using highly skilled manpower which the local people do not possess (Obi 2010). There is also the challenge arising from the low harmonization of project because of the complex procedure to negotiate and receive CDM approval, infrastructural challenge, finance and appropriate legislation which make CDM project implementation time consuming.

In particular, some CDM projects in Nigeria and indeed Africa are laughable and do not go far enough. For example, one of the CDM project - Efficient Fuel Wood Stoves (SAVE 80) for Nigeria involved the importation of 12,500 fuel efficient stoves from a Germany manufacturing company which is expected to save estimated meagre 31,309 tons of $\mathrm{CO}_{2}$ annually (UNFCCC 2006). It is not only disappointing, it is even more disproportionately that cooking stoves are to be imported from Germany in the name of CDM project when they can easily be manufactured locally in Nigeria. This is consistent with the views expressed by Liverman and Boyd (2008) that some set of projects like the wood stoves would come with a set of narratives about appropriate technology, poverty alleviation, gender and conversational which primarily benefit a few wealthy countries. There is also a huge question mark on how the wood stoves project concept meets the 
sustainable development, employment opportunities and technology transfer of Nigerians when they are imported from Germany.

Although the wood stoves is designed to use $80 \%$ less wood fuel than a traditional stove, substantially cutting user fuel costs, however, there are more questions about the prospects, efficacy and the rationale of clean energy technologies in Nigeria. In addition, the financial investments in small scale CDM projects are often insufficient to cover the overall high CDM transaction costs (Boyd 2009). Furthermore, Liverman and Boyd (2008); Lutken (2011) questioned the efficacy or otherwise of the CDM as an instrument for international cooperation on environmental sustainability which the world has embraced despite all its flaws and shortcomings, particularly when the benefit accruing to the LDCs are marginal. It therefore suffices to say that this is indeed a mechanism of dispossession and inequity.

\section{Conclusion}

This study seeks to offer a somewhat broader perspective on the realities versus the ideals of the CDM, particularly as it affects the LDCs. The reality is that there is inequitable distribution of projects to a few larger and more affluent developing countries which have fundamentally affected the original goal of the CDM as climate protection instrument. It is therefore, important, that the UNFCCC continue to explore alternative development model to attract investment to the LDCs because the investors are always in pursuit of an optimum investment portfolio. Investors are always looking in the direction of a more favourable climatic condition for their investment hence some countries tend to meet these criteria.

It is not enough to use technological and infrastructural development, enabling policy constraint, institutional capability and lack of legislative framework as the historical structural problem of the ideological and material foundations of capitalism concept which has enabled only very few countries to benefit from the CDM. The current structure of the CDM will have very little impact on the needs of LDCs towards sustainable development, adaptive capacity to sustainable development and climate change impacts. Thus, a radical shift in the distribution of CDM projects is critical to the economy and sustainable development needs of the LDCs as well as the long time survival of the CDM.

\section{Recommendations}

Based on the major shortcomings of the CDM on the uneven distribution of $\mathrm{CDM}$ projects, the following recommendations are made.

There is the need for continued cooperation to deepen the process of liberalization under a green capitalism that allows equitable distribution of CDM projects and to support the needs of the LDCs. Current climate change framework and policies need to adjust sustainably to accommodate the need of the LDCs where projects are distributed equitably. Therefore, to achieve the economic and social justice framework, the CDM need to build alternatives to capitalism's inexorable accumulation forces. 
There is a need for a critical review of the existing national regulatory framework, investment climate, legislation and institutional capability that hinders carbon credit investment and project finance in the LDCs. Adequate action has to be taken by the Parties to create stronger policies and modalities that address the development gap between the UNFCCC framework and the current state of project distribution in the CDM that is skewed in favour of few countries.

Finally, there should be a deliberate and regional effort towards equity, which may present a sound solution wherein synergies of countries within a region are created. A country quota for CDM project allocation may be counterproductive. However, a regional quota for the distribution of capacity development funds may be one solution towards this challenge that enables every country to benefit from the mechanism.

\section{References}

Alexeew, J., Bergset, L., Meyer, K., Petersen J., Schneider, L., Unger, C. (2010): An Analysis of the Relationship between the additionality of CDM Projects and their Contribution to Sustainable Development. International Environ Agreement, pg. 233 - 248.

Boyd E., Hultman, N., Roberts, J. T., Corbera, E., Cole, J., Bozmoski, A., Ebeling, J., Tippman, R., Mann, P., Brown, K. And Liverman, D. M. (2009): Reforming the CDM for Sustainable Development: Lessons Learned and Policy Future. Environmental Science and Policy Journal, pp. 820 - 831

Byigero, A. D., Clancy, J., Skutsch, M. (2010): CDM in Sub-Saharan Africa and the Prospects of the Nairobi Framework Initiative, Climate Policy, pg. 181 - 189.

Ellis, J. and Kamel, S. (2007): Overcoming Barriers to Clean Development Mechanism Projects, UNEP Riso Centre

Figueres, C. and Streck, C. (2009): The Evolution of the CDM in a Post-2012 Climate Agreement. The Journal of Environment and Development, pg. 18:227

Gupta, A. (2012): An Assessment of Success of Clean Development Mechanism of Kyoto Protocol in Climate Change Mitigation and Sustainable Development. Journal of Social Science \& Interdisciplinary Research Vol.1 Issue 8, pp. 42 - 52

Liverman, D. and Boyd, E. (2008): The CDM, Ethics and Development. In: A Reformed CDM - including new Mechanisms for Sustainable Development, Riso National Laboratory for Sustainable Energy, Copenhagen.

Lutken, S. E. (2011): Indexing CDM Distribution: Levelling the Playing Field, UNEP Energy Climate and Sustainable Development, Reso Centre.

Michaelowa, A., Jember, G. and Diagne E. M. (2014): Lessons from the CDM in LDCs, for the design of NMM and FVA

Michaelowa, Axel and Katharina Michaelowa. 2007. "Climate or Development: Is ODA Diverted from Its Original Purpose?” Climatic Change 84 (2007), pp. 5-21.

Obi, C. I. (2010): Oil Extraction, Dispossession, Resistance, and Conflict in Nigeria's Oil-Rich Niger Delta. Canadian Journal of Development Studies, 30

Olawuyi, D. S. (2010): From Kyoto to Copenhagen: Rethinking the Place of Flexible Mechanisms in the Kyoto Protocol's post 2012 Commitment Period. Law, Environment and Development Journal, p. 21.

Olsen, K. H. and Fenhann, J. (2008): A Reformed CDM - including new Mechanisms for Sustainable Development. In: United Nations Environment Programme, Riso National Laboratory for Sustainable Energy, Copenhagen

Olsen, Karen Holm. 2007. "The Clean Development Mechanism's Contribution to Sustainable Development: A Review of the Literature," Climatic Change 84 (2007), pp. 59-73.

Shishlov, I. and Bellassen, V. (2012): CDC Climate Report, 10 Lessons from 10 Years of the CDM.

Sovacool, B. K. (2011): Four Problems with Global Carbon Markets: A Critical Review. Journal of Energy and Environment, Vol. 22, No. 6, pg. 681-694 
Silayan, A. (2005): Equitable Distribution of CDM Projects among Developing Countries. Hamburgisches Welt-Wirtschafts-Archiv (HWWA), Hamburg Institute of International Economics, Hamburg

Sutter, C. and Parreno, J. C. (2007): "Does the Current Clean Development Mechanism Deliver its Sustainable Development Claim? An Analysis of Officially Registered CDM Projects," Climatic Change 84 (2007), pg. 75-90.

UNEP Riso Centre (2007): Enhancing a more Equitable Regional Distribution of CDM Project Activities. Overview of UNEP's CDM Activities

UNFCCC (2005): The Ovade Ogharefe Gas Capture and Processing Project. Clean Development Mechanism, Project Design Ducoment Form, UNFCCC, 20 October, 2005

UNFCCC (2006): Efficient Fuel Wood Stoves for Nigeria. http://cdm.unfccc.int/Projects/DB/RWTUV1245685309.5/view (accessed July 2014).

UNFCCC (2011): Benefits of the Clean Development Mechanism

UNFCCC (2012): Executive Board Annual Report

UNFCCC (2014): Promoting the CDM in Africa: Global carbon market outlook and regional context. Training Workshop on CDM Post-registration Changes (PRCs) and Programme of Activities (PoAs), Pretoria, South Africa

Watson, C. and Fankhauser, S. (2009): The Clean Development Mechanism: too Flexible to Produce Sustainable Development Benefits? Centre for Climate Change Economics and Policy.

Wood, B. T., Sallu, S. M. and Paavola, J. (2015): Can CDM Finance Energy Access in Least Developed Countries? Evidence from Tanzania. Sustainability Research Institute, School of Earth and Environment, University of Leeds, Leeds. 